\title{
A comparison of controlled ventilation with a noninvasive ventilator versus traditional mask ventilation
}

\author{
Mike Fogarty $^{1} \mathbb{D} \cdot$ Kai Kuck $^{2} \cdot$ Joseph Orr $^{2} \cdot$ Derek Sakata $^{3}$
}

Received: 12 December 2018 / Accepted: 17 July 2019 / Published online: 23 July 2019

(c) Springer Nature B.V. 2019

\begin{abstract}
After induction, but before intubation, many general anesthesia patients are manually bag-mask ventilated. The objective of this study was to determine the efficacy of bag-mask ventilation $(\mathrm{MkV})$ of an anesthetized patient versus mask ventilation using a noninvasive ventilator (NIV). We hypothesized that feedback-controlled, mask ventilation via NIV is more efficacious and safer. This critical short period of time in the operating room was chosen to compare MkV versus NIV. 30 ASA I-III patients, aged 18-74, presenting for elective surgery under general anesthesia were enrolled in the study. Patients were ventilated first with MkV and then with NIV. One minute of ventilation data was collected for each method. Respiratory inductance plethysmography (RIP) bands around the chest and abdomen were used to measure tidal volumes and breath rates for each method of ventilation. The NIV was set to deliver 10 breaths per minute with $12 \mathrm{cmH}_{2} \mathrm{O}$ of pressure support. A non-inferiority test was used to compare MkV and NIV. MkV breaths had an average of 13 breaths and tidal volume of $364 \mathrm{~mL}$ (SD $145 \mathrm{~mL}$ ). NIV resulted in an average of 10 breaths and tidal volume of $552 \mathrm{~mL}$, i.e., $188 \mathrm{~mL}$ more than MkV (lower bound of the $95 \%$ confidence interval equal to $120 \mathrm{~mL}$ ). The hypothesis of non-inferiority at the $-100 \mathrm{~mL}$ level and the superiority hypothesis at the $+100 \mathrm{~mL}$ level was accepted. NIV also resulted in much more consistent ventilation rates (zero variation since it is controlled by the ventilator) when compared to manual ventilation while maintaining safe airway pressures $\left(8 \mathrm{cmH}_{2} \mathrm{O}\right.$ EPAP and $20 \mathrm{cmH}_{2} \mathrm{O}$ IPAP). Feedback controlled mask ventilation via a NIV is a viable alternative to $\mathrm{MkV}$. It can deliver more optimal tidal volumes with the operator utilizing only one hand. The airway pressures are fixed at safe limits during a period where the goal is to reach a maximal level of oxygenation prior to intubation. Over-ventilation or over-pressurization of the airway is not a concern with NIV since the pressures are maintained well within safe thresholds to avoid injury.
\end{abstract}

Keywords Bag-valve-mask $\cdot$ Noninvasive ventilation $\cdot$ Mask ventilation $\cdot$ Bi-level positive pressure ventilation . Mechanical ventilation

\section{Introduction}

There are many situations during medical care that patients are manually ventilated $(\mathrm{MkV})$ using a bag-valve-mask (BVM) device such as emergencies (CPR), patient transport, and in between intubation attempts during general anesthesia. Manual ventilation requires one hand to secure the mask in place while holding the airway open and the other hand to squeeze the self-inflating bag. Over-pressuring and delivering overly rapid breaths during bag masking is very likely [1]. We hypothesize that mask ventilation,

Mike Fogarty

m.fogarty@utah.edu

Extended author information available on the last page of the article utilizing a noninvasive ventilator (NIV) utilizing automated feedback control, is more efficacious and safer than $\mathrm{MkV}$ using a BVM.

Manual ventilation using a BVM is a difficult technique to master and requires both hands of the person performing the ventilation as well as full attention to monitor for efficacy of ventilation $[2,3]$. Novice clinicians especially find it challenging to maintain an open airway while bag-mask ventilating patients. It is difficult to grasp the mask in a way that frees two fingers to provide the required chin-lift and the proper head-tilt to keep the airway open with one hand while squeezing the bag with the other hand. One hand is used to carefully squeeze the bag to ensure adequate tidal volume while being careful not to over-pressurize thereby causing gastric insufflation or barotrauma. If the airway is 
obstructed, the clinician, who is squeezing the bag, may mistakenly think he/she is delivering an adequate tidal volume, when in fact the entire tidal volume may be lost through a leak between the mask and the patient's face [4]. Achieving adequate bag-mask ventilation skills is so challenging, yet so critical, that the literature calls for additional training of medical staff in order to raise consistency and avoid complications associated with mask ventilation [5-9]. Because this task requires the full attention of the person giving ventilation, a second clinician is needed to perform additional patient care tasks such as administering medications and providing other care.

Further, it is difficult for clinicians to provide a consistent respiratory rate during mask ventilation. Clinical studies indicate that even trained clinicians give on average 25-35 breaths per minute, compared to the $10-12$ breaths per minute prescribed by ACLS guidelines [10, 11]. Minimizing breath rate is difficult because delivering artificial respiration, especially under adverse clinical conditions, alters time perception [4]. Successful mask ventilation is classified as approximately $8-10 \mathrm{~mL} / \mathrm{kg}$ (ideal body weight) tidal volume and an upper limit pressure of $20-25 \mathrm{cmH}_{2} \mathrm{O}[12,13]$.

Ensuring adequate ventilating pressures, to achieve therapeutic tidal volumes during manual mask ventilation, is also difficult. Excessive pressures can cause a decrease in cardiac preload, traumatic brain injury, hemorrhagic shock, gastric insufflation [14-16], and lung injury. However, insufficient pressures can lead to inadequate ventilation and concomitant hypoxia and hypercapnia. Difficult or impossible mask ventilation in respiratory compromised patients is a medical emergency. The incidence of difficult or impossible mask ventilation is reported as $1.4 \%$ in adult patients undergoing general anesthesia [17-20].

Noninvasive ventilation (NIV) is becoming a commonly accepted way to maintain ventilation in respiratory compromised patients in the ICU. This method avoids using invasive airway devices such as endotracheal tubes and instead supports a compromised airway with a pneumatic splint of air pressure. Compared with intubation, NIV reduces the length of intensive care unit (ICU) and hospital stay, morbidity, and mortality in patients with acute and chronic respiratory failure [21].

The objective of this study was to compare delivered ventilation by an experienced anesthesia provider using an NIV device (V60, Phillips-Respironics, Carlsbad, CA) versus MkV using a standard BVM device. The Respironics V60 Ventilator is an assist ventilator and is intended to augment patient breathing by applying CPAP and bi-level positive airway pressure. It is intended for spontaneously breathing individuals who require mechanical ventilation support. Patients undergoing general anesthesia are often induced and paralyzed in preparation for intubation. After induction, but before intubation, many patients are manually bag-mask ventilated $(\mathrm{MkV})$. This highly controlled period of time was chosen to compare the two methods of ventilation. We hypothesized that NIV would ventilate with equal or greater tidal volumes than manual ventilation by experienced anesthesia providers. This can be accomplished with safe airway pressures and more consistent breath rates, because NIV inherently limits patient airway pressures within safe thresholds (less than $20 \mathrm{cmH}_{2} \mathrm{O}$ ) and controls rate at which breaths are delivered.

\section{Methods}

Following IRB approval and informed consent, 30 ASA status I-III patients, aged 18-74, presenting for elective surgery requiring general anesthesia at the University of Utah, John Moran Eye Center were enrolled in the study. A sample size of 30 patients was predicted to have $95 \%$ power to detect the non-inferiority of noninvasive ventilation compared to $\mathrm{MkV}$. This a priori power analysis was based on best predictions by experienced clinicians. This study was registered with ClinicalTrials.gov on 12/4/2015. The responsible party and principal investigator is Dr. Kai Kuck, Director of Bioengineering, Department of Anesthesiology at the University of Utah. The registration number is NCT02623270.

Exclusion criteria for patients, included oropharyngeal or facial pathology, risk of aspiration (defined by need for rapid sequence intubation, uncontrolled gastroesophageal reflux disease), known and/or documented difficulty placing an endotracheal tube in the past, limited neck extension or flexion, restrictive lung disease, personal or familial history of malignant hyperthermia, and known or predicted severe respiratory disease or compromise. Female subjects must have had a negative urine pregnancy screen.

Prior to entering the operating room, the patient was fitted with respiratory inductance plethysmography (RIP) bands placed around the chest and abdomen (Model 0570, Braebon Medical, Ontario, Canada). These bands measure the relative change in volume of the thorax and abdomen. During breathing, these changes equate to signals that are proportional to the volume of air exchanged by the lungs. After pre-oxygenation, induction of anesthesia and adequate neuromuscular blockade (train of four less than 1 out of 4), noninvasive ventilation and manual anesthesia bag ventilation, in this order, were used to ventilate each enrolled patient for $1 \mathrm{~min}$ each. Tracheal intubation commenced immediately after both masking tests were completed. The experienced provider held the mask in position and the noninvasive ventilator was used to ventilate the patients. For patient safety, and to avoid gastric insufflation (which would increase the risk of aspiration), inspiratory pressures during ventilation were limited to $20 \mathrm{cmH}_{2} \mathrm{O}$. The signals from the RIP bands were 
continuously monitored during the study and during the first 2 min after the patient had been intubated and ventilated by the anesthesia machine.

The relationship between RIP signal and breath (tidal) volume varies depending on patient size, band placement, patient position, etc. The direct measurement of patient tidal volume and the corresponding RIP signals measured post-intubation were used to obtain calibration factors so that tidal volume could be calculated from the RIP signals obtained during the pre-intubation phase of the study. The relative volume of breaths given by the noninvasive ventilator were measured. The data collected and recorded on the portable computer included age, height, weight, BMI, OSA status, RIP data, mask pressure, flow, and breath rate.

Hemodynamic variables including blood pressure, heart rate, and pulse oximetry were monitored during this time as part of routine clinical monitoring. Presence of ventilation was clinically assessed as chest rise and fall and end tidal carbon dioxide measurement. Additionally, oxygen saturation levels were maintained above $90 \%$. If, at any point during the study, ventilation became inadequate (i.e., loss of chest rise, loss of end tidal carbon dioxide signal, oxygen saturation $<90 \%$ ), the attending anesthesiologist took control of the airway to regain adequate ventilation.

\section{Study procedures}

\subsection{Induction of anesthesia}

Patients underwent a standard of care anesthesia protocol. Antiemetics, vasopressors, or blood pressure lowering medications were given as needed at the discretion of the attending anesthesiologist as standard of care in the operating room.

\subsection{After induction}

Initially, an oral airway was not used. One patient in this study required the use of an oral airway and the same one was used for both methods of ventilation. Once a train-offour less than 1 out of 4 was obtained, to ensure adequate muscle relaxation, the experienced anesthetist was asked to manually ventilate the patient as he or she normally would. During manual ventilation, the oxygen flowed through the standard breathing circuit with the fresh gas flow rate set at $10 \mathrm{~L} / \mathrm{min}$ and the patient was ventilated. The anesthetist ventilated the patient until he felt comfortable that the patient could be ventilated adequately (average of $1.01 \mathrm{~min}$ and 12.77 breaths per patient).

\subsection{Testing}

The noninvasive ventilator was set to deliver 10 breaths per minute with Bi-level positive airway pressure settings of $20 \mathrm{cmH}_{2} \mathrm{O}$ during inspiration (1 s inspiratory time) and $8 \mathrm{cmH}_{2} \mathrm{O}$ during exhalation. All other modes and algorithms included on the ventilator were disabled and it simply regulated the pressure to deliver breaths at the set rate. The inspired oxygen fraction given by the ventilator was set at the maximum level (100\% oxygen). The amount of air placed in the face cushion of the bag-valve masks was standardized at $150 \mathrm{cc}$. During ventilation with the noninvasive ventilator, the provider was instructed to hold the mask on the patient's face with one hand and to use their standard grip on the mask. If this was not sufficient, the provider moved to a two-handed mask ventilation. Once satisfied with ventilation delivery, the ventilator was set to deliver 10 consecutive breaths at a set rate of 10 breaths per minute. The clinician was notified by the noninvasive ventilator if an insufficient volume of gas reached the patient's lungs. After 10 consecutive breaths were delivered, the anesthetist proceeded to intubate the patient.

\subsection{After intubation}

After intubation, tidal volume measurements by an on-airway flow sensor connected to a respiratory profile monitor (NM3, Philips Medical, Carlsbad, CA) were recorded as simple inspiratory and expiratory flow values along with the simultaneously collected RIP signals for $2 \mathrm{~min}$. The $\min / \max$ values of the RIP signals were calibrated using the summed tidal volumes measured by the flow sensor for each breath.

\subsection{Intraoperative and postoperative care}

Following the data collection for calibration purposes, the flow sensor was removed from the breathing circuit. The RIP bands were removed from the patient and anesthesia care was provided by, and at the discretion of, the anesthesiologist and or anesthetist as deemed appropriate. The patient was routinely awakened from anesthesia and transported to the PACU to receive postoperative attention according to standard of care.

\subsection{Data analysis}

Before analysis, an investigator removed any identification of the patients. Statistical analysis was performed for noninferiority during $\mathrm{MkV}$ and noninvasive ventilation to show that NIV can provide equal or better ventilation than the current standard of care using tidal volume as the variable to judge quality of ventilation. The noninferiority analysis was 
modeled after the analysis Rossignol et al. performed [22]. The noninferiority threshold was specified as $-1 \mathrm{~mL} / \mathrm{kg}$ average delivered tidal volume. Additionally, a superiority criteria was set at $+1 \mathrm{~mL} / \mathrm{kg}$ average delivered tidal volume based on the clinical expertise of collaborating anesthesiologists and current ventilation guidelines $(6-8 \mathrm{~mL} / \mathrm{kg})[23$, 24].

\section{Results}

Noninvasive ventilation resulted in higher tidal volumes delivered to the patient when compared to $\mathrm{MkV}$ via bagmask ventilation (MkV: $364 \mathrm{~mL}$ [95\% CI 320-407 mL] vs. NIV: $552 \mathrm{~mL}$ [95\% CI $484-620 \mathrm{~mL}$ ], $\mathrm{P}=0.005$. NIV was also greater when tidal volume was converted to $\mathrm{mL} / \mathrm{kg}$ (ideal body weight) (MkV: $6.0 \mathrm{~mL} / \mathrm{kg}$ [95\% CI $5.4-6.7 \mathrm{ml} / \mathrm{kg}$ ] vs. NIV: $9.2 \mathrm{~mL} / \mathrm{kg}$ [95\% CI $8.1-10.2 \mathrm{~mL} /$ $\mathrm{kg}$ ], $\mathrm{P}=0.005$ ), with a difference between the two methods of $3.2 \mathrm{~mL} / \mathrm{kg}$, with a $2.1 \mathrm{~mL} / \mathrm{kg}$ lower bound of the $95 \%$
CI. That value is greater than the noninferiority threshold that was specified as $-1 \mathrm{~mL} / \mathrm{kg}$ thus demonstrating the noninferiority hypothesis at the $5 \%$ error level. The superiority threshold was set at $+1 \mathrm{~mL} / \mathrm{kg}$ and was reached (Fig. 1).

Noninvasive ventilation resulted in much more consistent ventilation rates when compared to manual ventilation via bag-mask (MkV: 13 breaths/min [95\% CI 11.9-14.0 breaths/ $\mathrm{min}]$ vs. NIV: 10 breaths/min with zero variation, $\mathrm{P}=0.005$ ).

Standard deviations were used on a per breath basis to evaluate the consistency of ventilation delivered with both methods. Since each patient is physiologically different, a standard deviation was calculated for each method for each patient. This allows a comparison of the consistency of the ventilation per patient instead of across the entire population. NIV resulted in much more consistent tidal volumes when compared to manual ventilation as shown by these standard deviations (MkV: $91.2 \mathrm{SD}(\mathrm{mL})$ [95\% CI 69.6-112.7 SD (mL)] vs. NIV: 58.9 SD (mL) [95\% CI 49.0-69.0], $\mathrm{P}=0.005$ ) (Fig. 2). The smaller standard deviations and
Fig. 1 Inferiority and superiority analysis of $\mathrm{MkV}$ versus NIV

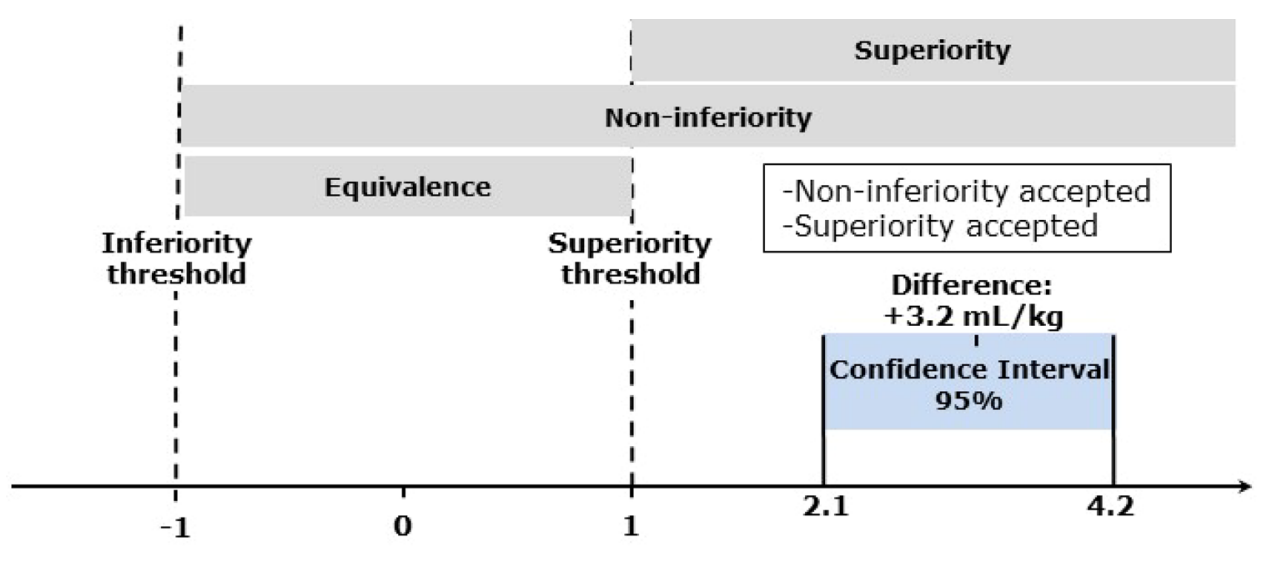

Difference in Tidal Volume: NIV - BVM ( $\mathrm{mL} / \mathrm{kg})$

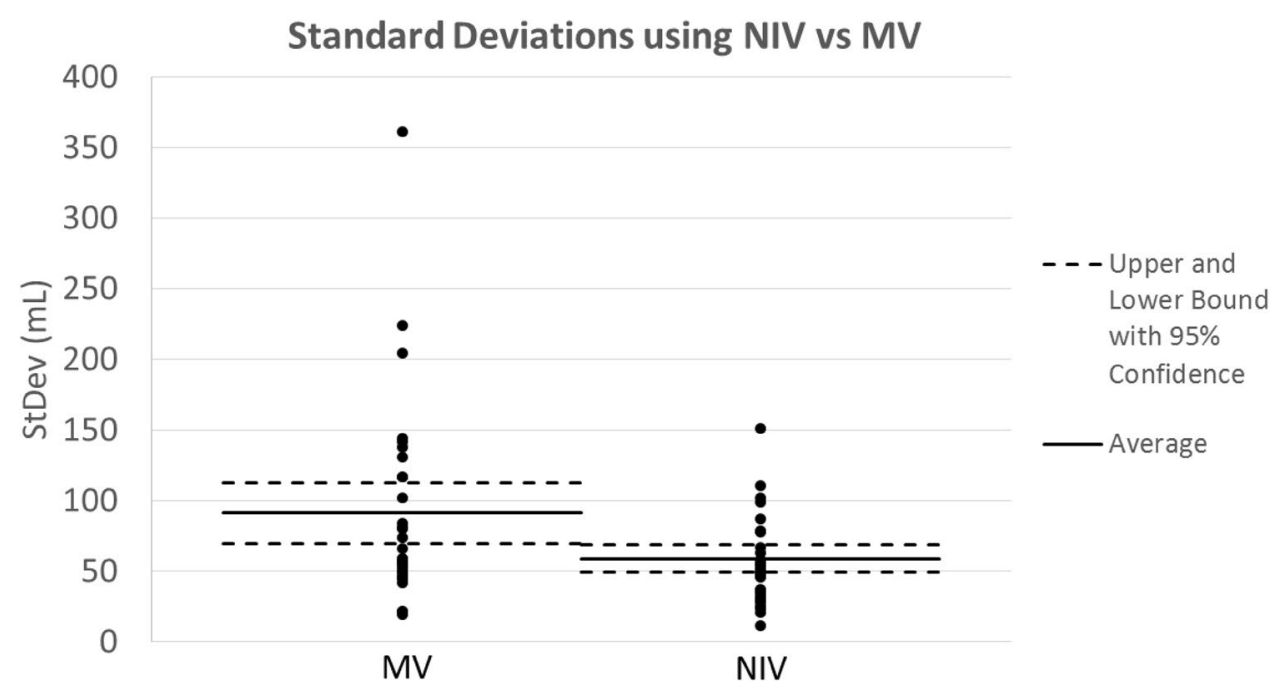

Fig. 2 Variability of tidal volumes delivered by $\mathrm{MkV}$ versus NIV 
more narrow spread observed with noninvasive ventilation imply more consistent ventilation delivered to the patients.

\section{Conclusions}

The objective of this study was to compare delivered ventilation by an experienced anesthesia provider using an NIV device versus MkV using a standard BVM device. The endpoint of the study was evaluated by way of a non-inferiority/ superiority analysis between the standard of care $\mathrm{MkV}$ and automated NIV. The study was motivated by the desire to develop a portable, easy-to-use, noninvasive ventilator that would be able to replace BVM devices and provide superior ventilation and monitoring (breath rate, tidal volume, and airway pressures) during patient sedation, transport, and other intervals where a patient's airway may become compromised. The interval of anesthesia utilized in this study is not meant to be the target for usage of such a ventilator. It is understood that for such a brief period of time in the operating room, it might not be feasible to add a ventilator to the mix. However, this study utilized the controlled nature of this period of sedation to analyze and compare these two methods of ventilation so that the principles could be applied to other areas of patient care that might require them. The clinical relevance of the study's objective can be broken down into three main sections: improved oxygenation through higher tidal volumes, patient safety by way of limiting pressures introduced into the lungs, and consistency of the ventilation technique.

NIV resulted in significantly higher delivered tidal volumes with a set safe upper pressure limit when compared to the standard of care MkV. The priority for this type of ventilation, immediately following induction and preceding intubation, is to maximize alveolar ventilation and thus oxygenation, while still maintaining airway pressures below the safe threshold so as to avoid lung injury. Optimal oxygenation delays the onset of hypoxia in an apneic patient. In the case of a difficult or impossible intubation, this can extend the time it takes for the patient to desaturate by up to 8 min [25]. The standard of care is a BVM device manually controlled by the clinician to oxygenate the patient and prepare them for endotracheal intubation. Increased alveolar ventilation will lead to higher oxygen concentrations within the patient's lungs. The data in this study demonstrates that it is possible to achieve optimal alveolar ventilation with set, safe upper pressure limits during this period using a feedback-controlled ventilator (Fig. 3). Additionally, it shows that noninvasive positive pressure ventilation is possible on an apneic patient by simply holding the mask in place as opposed to standard NIV machines that require mask fittings to minimize and keep leak constant.
BVM vs. V60

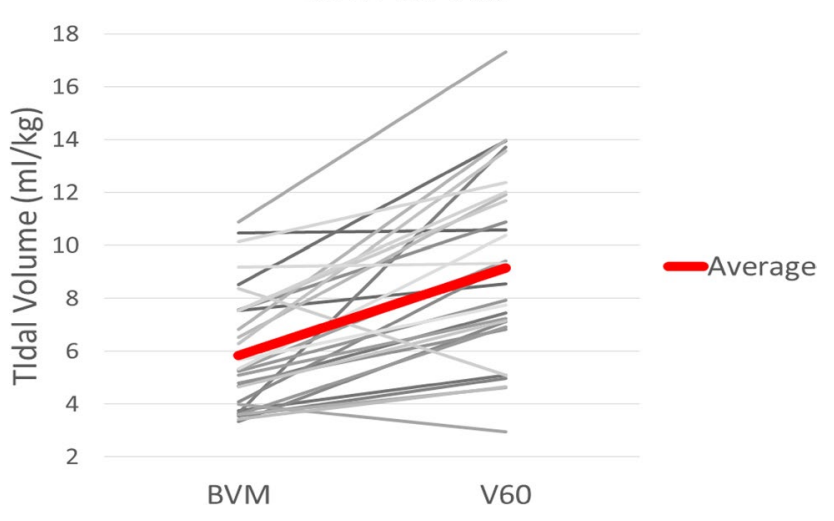

Fig. 3 Tidal volume comparison between MkV and NIV. Each line represents a single patient in the study

Automatic, feedback controlled NIV excels beyond the capabilities of manual ventilation in its ability to precisely control the pressure within the airway. Over pressurization of the airway poses a variety of risks to the patient. The simplest of these risks is barotrauma which causes actual physical injury to the lungs. Another risk is stomach insufflation and associated risks such as hypoventilation and increased risk of aspiration of stomach contents. Multiple studies have reported on the risks and prevalence of stomach insufflation and recommend that peak airway pressures be limited to approximately $20 \mathrm{cmH}_{2} \mathrm{O}$ [26-28]. This study did not collect or analyze pressure data during either ventilation mode. However, it is important to highlight this difference between $\mathrm{MkV}$ and NIV. Bi-level positive airway pressure NIV controls the pressures within the airway and therefore can mitigate barotrauma and/or stomach inflation. During $\mathrm{MkV}$, especially during emergency situations, the clinician/ rescuer is inclined to not only give more breaths/min [29] but also more forcefully compress the bag to increase the flow of gas into the patient's lungs. Both of these circumstances can lead to over pressurization of the lungs $[1,9]$.

In recent years there has been an ongoing debate regarding lung protective ventilation by way of low tidal volume mechanical ventilation, and whether it is appropriate for all patients [30, 31]. There are two main mechanisms for biophysical lung injury during mechanical ventilation. The first is overdistension of the lungs and the second is cell damage associated with the cyclic recruitment and derecruitment of unstable units within the lung. NIV avoids both of these modes of injury. NIV limits the pressures to safe ranges, therefore avoiding over pressurization and overdistension of the lungs. Additionally, NIV utilizes positive end expiratory pressure (PEEP) during exhalation, therefore avoiding the collapse of unstable units within the lung. NIV mitigates both of the mechanisms of lung injury during mechanical ventilation. The main objective of artificial ventilation is 
always to maximize gas exchange to the patient's lungs, thereby maximizing oxygen delivered to the lungs and elimination of $\mathrm{CO}_{2}$. The higher the alveolar ventilation is, the better that goal can be achieved. In our study, employing NIV, better alveolar ventilation was accomplished while also employing lung protective ventilation $\left(<20 \mathrm{cmH}_{2} \mathrm{O}\right)$.

$\mathrm{MkV}$ is not consistent in the breath rate, tidal volumes or airway pressures delivered $[4-9,12]$. These problems are especially evident during difficult masking situations. Difficult mask ventilation occurs between 1.4 and 5\% of the time depending on the criteria used for definition [32]. Difficult mask ventilation occasionally requires the use of a two-handed technique that requires an additional person to compress the bag. These problems led us to develop a portable, easy-to-use, electric blower-based ventilator that would effectively combine the simplicity and portability of a BVM with the precision and high-tech monitoring of NIV ventilators. Current NIV machines are generally too bulky and expensive for most applications outside of the ICU that the BVM occupies.

Current noninvasive ventilation, most commonly in the ICU, is administered via a mask held in place by straps. Before ventilation can begin the mask and straps are fitted to the patient to minimize air leaks. The minimal and consistent air leaks allow for accurate calculation of ventilation parameters by the ventilator being used. This study examined the performance of such a ventilator while the mask was held in place manually by the clinician using a standard one-handed grip on the mask. Since the ventilation parameters were measured external to the ventilator, we were able to evaluate the performance of breath delivery in the face of constantly varying leak conditions. The ventilator in development is capable of gathering accurate breath data even with variable leak. Such a ventilating device would cover patient transport, emergency situations, and essentially any time that a standard BVM is used. Current usage of BVM is widespread because its simplicity and low cost. Up until recent technological advancements, NIV technology was far too expensive to be considered as a replacement for the BVM. However, NIV has clear advantages for patient safety over manual ventilation with the BVM. As the price for this technology continues to drop it is important to evaluate where it fits into the healthcare economy.

As a small localized study, there were some limitations to our work that can be addressed in future work. Only three different experienced providers were used during the study, which likely led to more interpatient consistency of manual ventilation than would occur in a real world setting and was observed in other studies. A wider variety of providers and surgical centers would provide a better picture of the differences and similarities between these modes of ventilation. A further expansion of this study would be to independently measure the pressures within the patient's airway during
MkV and mask ventilation with NIV. Other studies have performed these measurements independently but a direct comparison would be best.

This study determined that NIV could ventilate with better tidal volumes than $\mathrm{MkV}$ by experienced anesthesia providers. This was accomplished with safe airway pressures and more consistent breath rates, because NIV inherently limits patient airway pressures within safe thresholds (less than $20 \mathrm{cmH}_{2} \mathrm{O}$ ) and controls rate at which breaths are delivered. Both non-inferiority and superiority was achieved when comparing standard $\mathrm{MkV}$ and noninvasive ventilation. This data paves the way for a novel portable, easy-to-use, noninvasive ventilator to be further studied and used during times where a $\mathrm{MkV}$ is the standard of care.

Funding Funding was provided by Utah Space Grant Consortium.

\section{Compliance with ethical standards}

Conflict of interest The authors declare that they have no conflict of interest.

Ethical approval All procedures performed in studies involving human participants were in accordance with the ethical standards of the institutional and/or national research committee and with the 1964 Helsinki declaration and its later amendments or comparable ethical standards. This article does not contain any studies with animals performed by any of the authors.

\section{References}

1. Von Goedecke A, Voelckel WG, Wenzel V, et al. Mechanical versus manual ventilation via a face mask during the induction of anesthesia: a prospective, randomized, crossover study. Anesth Analg. 2004. https://doi.org/10.1213/01.ANE.0000096190.36875 67.

2. Baskett PJF, Bossaert L, Carli P, et al. Guidelines for the basic management of the airway and ventilation during resuscitation. A statement by the airway and ventilation management working group of the European resuscitation council. Resuscitation. 1996;31:187-200.

3. Baskett PJF, Bossaert L, Carli P, et al. Guidelines for the advanced management of the airway and ventilation during resuscitation. A statement by the airway and ventilation management of the working group of the European resuscitation council. Resuscitation. 1996;31:201-30.

4. Cooper JA, Cooper JD, Cooper JM. Cardiopulmonary resuscitation: history, current practice, and future direction. Circulation. 2006;114:2839-49. https://doi.org/10.1161/CIRCULATIO NAHA.106.610907.

5. Brinker A, Stratling WM, Schumacher J. Evaluation of bag-valvemask ventilation in simulated toxic environments. Anaesthesia. 2008;63:1234-7. https://doi.org/10.1111/j.1365-2044.2008.05579 .x.

6. Gurajala I, Azharuddin M, Gopinath R. General anaesthesia with laryngeal mask airway may cause recurrence of pneumocephalus in a patient with head injury. Br J Anaesth. 2013;111:675-6. https ://doi.org/10.1093/bja/aet316. 
7. Soleimanpour H, Gholipouri C, Panahi JR, et al. Role of anesthesiology curriculum in improving bag-mask ventilation and intubation success rates of emergency medicine residents: a prospective descriptive study. BMC Emerg Med. 2011;11:8. https:// doi.org/10.1186/1471-227X-11-8.

8. Sollid SJ, Heltne J, Søreide E, Lossius H. Pre-hospital advanced airway management by anaesthesiologists: is there still room for improvement? Scand J Trauma Resusc Emerg Med. 2008;16:2. https://doi.org/10.1186/1757-7241-16-2.

9. De Regge M, Vogels C, Monsieurs KG, Calle PA. Retention of ventilation skills of emergency nurses after training with the SMART BAG ${ }^{\circledR}$ compared to a standard bag-valve-mask. Resuscitation. 2006;68:379-84. https://doi.org/10.1016/j.resuscitat ion.2005.07.013.

10. Abella BS, Alvarado JP, Myklebust H, et al. Quality of cardiopulmonary resuscitation during in-hospital cardiac arrest. JAMA. 2005;293:305-10. https://doi.org/10.1001/jama.293.3.305.

11. Aufderheide TP. Hyperventilation-induced hypotension during cardiopulmonary resuscitation. Circulation. 2004;109:1960-5. https://doi.org/10.1161/01.CIR.0000126594.79136.61.

12. Golzari SE, Soleimanpour H, Mehryar H, et al. Comparison of three methods in improving bag mask ventilation. Int J Prev Med. 2014;5:489-93.

13. Balk RA. Setting the tidal volume in adults receiving mechanical ventilation: lessons learned from recent investigations. 2017. https://renew.nbrc.org/Documents/Tidal\%20Volume\%20Settings $\% 20$ in\%20Adult\%20Mechanical\%20Ventilation.pdf.

14. Smally AJ, Ross MJ, Huot CP. Gastric rupture following bagvalve-mask ventilation. J Emerg Med. 2002;22:27-9.

15. Dörges V, Wenzel V, Knacke P, Gerlach K. Comparison of different airway management strategies to ventilate apneic, nonpreoxygenated patients. Crit Care Med. 2003;31:800-4. https://doi. org/10.1097/01.CCM.0000054869.21603.9A.

16. Meier C. Airway management in patients with brain injury. Emerg Nurse J RCN Accid Emerg Nurs Assoc. 2013;21:18-23. https:// doi.org/10.7748/en2013.12.21.8.18.e1236.

17. Shah P, Sundaram V. Incidence and predictors of difficult mask ventilation and intubation. J Anaesthesiol Clin Pharmacol. 2012;28:451. https://doi.org/10.4103/0970-9185.101901.

18. Airway AS of ATF on M of the D. Practice guidelines for management of the difficult airway. Anesthesiology. 1993;78:597-602.

19. Kheterpal S, Han R, Tremper KK, et al. Incidence and predictors of difficult and impossible mask ventilation. Anesthesiology. 2006;105:885-91.

20. Apfelbaum JL, Hagberg CA, Caplan RA, et al. Practice guidelines for management of the difficult airway: an updated report by the american society of anesthesiologists task force on management of the difficult airway. Anesthesiology. 2013;118:251-70. https:// doi.org/10.1097/ALN.0b013e31827773b2.

21. Carron M, Freo U, BaHammam AS, et al. Complications of non-invasive ventilation techniques: a comprehensive qualitative review of randomized trials. Br J Anaesth. 2013;110:896-914.

22. Rossignol P, Dorval M, Fay R, et al. Rationale and design of the HepZero study: a prospective, multicenter, international, open, randomized, controlled clinical study with parallel groups comparing heparin-free dialysis with heparin-coated dialysis membrane (Evodial) versus standard care: study protocol for a randomized controlled trial. Trials. 2013;14:163. https://doi. org/10.1186/1745-6215-14-163.

23. Ferguson ND. Low tidal volumes for all? JAMA. 2012;308:1689. https://doi.org/10.1001/jama.2012.14509.

24. Nava Stephano, Fanfulla Francesco. Non-invasive artificial ventilation: how, when and why. Milan: Springer; 2013.

25. Frerk C, Mitchell VS, McNarry AF, et al. Difficult airway society 2015 guidelines for management of unanticipated difficult intubation in adults. Br J Anaesth. 2015;115:827-48. https://doi. org/10.1093/bja/aev371.

26. Weiler N, Heinrichs W, Dick W. Assessment of pulmonary mechanics and gastric inflation pressure during mask ventilation. Prehospital Disaster Med. 1995;10:101-5.

27. Melker RJ, Banner MJ. Ventilation during CPR: two-rescuer standards reappraised. Ann Emerg Med. 1985;14:397-402.

28. Von Goedecke A, Wenzel V, Hörmann C, et al. Effects of face mask ventilation in apneic patients with a resuscitation ventilator in comparison with a bag-valve-mask. J Emerg Med. 2006;30:637. https://doi.org/10.1016/j.jemermed.2005.02.021.

29. Milander MM, Hiscok PS, Sanders AB, et al. Chest compression and ventilation rates during cardiopulmonary resuscitation: the effects of audible tone guidance. Acad Emerg Med. 1995;2:708-13.

30. Hubmayr RD. Point: is low tidal volume mechanical ventilation preferred for all patients on ventilation? Yes. Chest. 2011;140:911. https://doi.org/10.1378/chest.11-0825.

31. Md LG. Counterpoint: is low tidal volume mechanical ventilation preferred for all patients on ventilation? No. Chest. 2011;140:113. https://doi.org/10.1378/chest.11-0827.

32. El-Orbany M, Woehlck HJ. Difficult mask ventilation. Anesth Analg. 2009;109:1870-80. https://doi.org/10.1213/ANE.0b013 e3181b5881c.

Publisher's Note Springer Nature remains neutral with regard to jurisdictional claims in published maps and institutional affiliations.

\section{Affiliations}

\section{Mike Fogarty $^{1}$ (D) Kai Kuck ${ }^{2} \cdot$ Joseph Orr $^{2} \cdot$ Derek Sakata $^{3}$}

Kai Kuck

kai.kuck@hsc.utah.edu

Joseph Orr

joseph.orr@hsc.utah.edu

Derek Sakata

Derek.Sakata@hsc.utah.edu

1 Departments of Anesthesiology and Bioengineering, University of Utah School of Medicine, 30 North 1900 East, Room 3C444, Salt Lake City, UT 84132, USA
2 Department of Anesthesiology, University of Utah School of Medicine, 30 North 1900 East, Room 3C444, Salt Lake City, UT 84132, USA

3 Department of Anesthesiology, University Hospital Anesthesiology, 50 North Medical Dr., Salt Lake City, UT 84132, USA 